\title{
BER performance analysis of OFDM-MIMO system using GNU Radio
}

\author{
M.Ushamahesh Singh ${ }^{1 a}$, Sushil Kakkar ${ }^{2}$ and Shweta Rani ${ }^{3}$ \\ Electronics and Communication Engineering Department, BGIET, Punjab, India
}

\begin{abstract}
Multiple Input Multiple Output (MIMO) channels can be used to increase the data rate and the channel capacity by employing multiple transmitting and receiving antennas at both the ends of a wireless communication system. MIMO systems employ Orthogonal Frequency Division Multiplexing (OFDM) technique and it uses separate antennas at both the transmitter and receiver to increase the data rate and with OFDM, instead of a single carrier, the main information is modulated into a number of independent sub-carrier signals which are orthogonal to each other. This paper presents an OFDM-MIMO transceiver design and the performance analysis of the system based on Error rate for different modulation techniques using GNU Radio. OFDM is chosen over a single carrier solution due to lower complexity of equalizers for high delay spread channels or high data rates. So the combination of MIMOOFDM system has become a potential technology for high speed data transmission and efficient utilization of the channel spectrum for the modern wireless communication networks.
\end{abstract}

\section{Introduction}

Due to the sharp increase in demand for wireless connectivity during the last two decades, the performance assessment of wireless communication systems are the current area of research. Some of the challenges that the wireless technology has faced includes signal fading, multipath, limited frequency spectrum. The number of users are increasing and the service providers are using limited bands and there are still wasted and unused frequencies due to different factors and parameters. The wireless communication systems are not as reliable as guided medium communication mainly due to fading and other propagation effects and accordingly, techniques to improve its capacity and reliability has become one of the most important area of current research. It is shown that by using the technique of multiple antennas at both the transmitting and receiving ends, the high performance $4 \mathrm{G}$ broadband wireless is enabled. Such a wireless technology is called Multiple Input Multiple Output. MIMO systems employ Orthogonal Frequency Division Multiplexing to achieve higher throughput without the extension of the bandwidth available. So for the next generation wireless communication systems such as WiMAX, LTE, WLAN, WMAN standards which accommodate many users in the same channel, the combination of MIMO-OFDM technique is considered as the best technique that overcome many challenges of today's wireless technology and it is seemed to be a solution for the future broadband wireless systems. Further, the MIMO-OFDM meets the requirements of high data rate and high capacity without the need of extending the available spectrum. While studying MIMO technology and OFDM technique, this paper sets up a

\footnotetext{
${ }^{\mathrm{a} C o r r e s p o n d i n g ~ a u t h o r: ~ p i t t e r k y m @ g m a i l . c o m ~}$
}

simple MIMO-OFDM wireless communication system and the performance is evaluated using the GNU radio.

\section{GNU Radio}

The system presented in this paper is accomplished using GNU radio which is an evolving free and open source software based on the concept of blocks and flow graphs. It is a powerful SDR software that contains a set of easily reconfigurable blocks and can be easily adapted to different network architectures and protocols. Ranging from easily reconfigurable blocks, it has blocks with different parameters that are used in most of the recent wireless communication technologies in which when connected together forms transmitter and receiver chains. The GNU radio applications are primarily written using python language and the performance critical signal processing blocks are implemented in $\mathrm{C}++$. It provides a library of signal processing blocks which are written in $\mathrm{C}++$ language that implement various functionalities of both the transmit and receive architectures. These blocks can be used and combined by the final user through Python. GNU radio provides a Python interface for each $\mathrm{C}++$ blocks as well as tools to connect various blocks. In this way, the simplified wrapper and interface generation allows $\mathrm{C}++$ code to be used from Python. Blocks are basic operation units that process continuous data streams and each block has a number of input and output ports.

\section{MIMO and OFDM}

\subsection{MIMO Technology and system model}


The Multiple Input Multiple Output (MIMO) employs multiple antennas in the transmitter and receiver at the same time of the communication system and it uses the random fading and multipath propagation to increase its transmission rate. MIMO provides transmit diversity and receiver diversity. It can be used in different kind of networks like PAN, MAN, WAN, WLAN, LAN and can receive either a diversity gain, capacity gain and to overcome signal fading. A MIMO system has three main components mainly transmitter, channel and receiver. The function of the transmitter is to send multiple data such as $\mathrm{X}_{1}, \mathrm{X}_{2}, \mathrm{X}_{3}, \ldots, \mathrm{X}_{\mathrm{N}}$ using the different transmit antennas, $N_{t}$ and the signals $R_{1}, R_{2}, R_{3}, \ldots, R_{N}$ are received by each receive antennas, $\mathrm{Nr}$ simultaneously. If at a certain time, the complex signals $\mathrm{X}_{1}, \mathrm{X}_{2}, \mathrm{X}_{3}, \ldots \ldots, \mathrm{X}_{\mathrm{N}}$ are transmitted via $\mathrm{N}_{t}$ transmit antennas, then the received data $R_{1}, R_{2}, R_{3}, \ldots, R_{N}$ for $\mathrm{Nr}$ received antennas can be expressed as[13]

$$
\begin{gathered}
R_{l}=h_{11} X_{1}+h_{12} X_{2}+\ldots .+h_{1 N} X_{N} \\
R_{2}=h_{21} X_{1}+h_{22} X_{2}+\ldots .+h_{2 N} X_{N} \\
\ldots \ldots \\
R_{N}=h_{N 1} X_{1}+h_{N 2} X_{2}+\ldots .+h_{N N} X_{N}
\end{gathered}
$$

By combining all the received signals in a vector $\mathrm{R}$ the MIMO signal model is described as

$$
R=H X+n
$$

Where $\mathrm{R}$ is $\mathrm{Nr} \times 1$ received signal vector, $\mathrm{H}$ is $\mathrm{Nt} \times 1$ transmitted vector and $\mathrm{n}$ is $\mathrm{Nr} \times 1$ Gaussian noise vector. With $\mathrm{Nt}$ inputs and $\mathrm{Nr}$ outputs the channel can be expressed as $\mathrm{Nr} \times \mathrm{Nt}$ MIMO channel transfer matrix $\mathrm{H}$, given by [13]

$$
\mathrm{H}=\left[\begin{array}{cccc}
h_{11} & h_{12} & \ldots & h_{1 N t} \\
h_{21} & h_{22} & \ldots & h_{2 N t} \\
\vdots & \vdots & \vdots & \vdots \\
h_{N 1} & h_{N 2} & \ldots & h_{N r N t}
\end{array}\right]
$$

where hij is the attenuation and phase shift between the $\mathrm{j}^{\text {th }}$ transmitter and $\mathrm{i}^{\text {th }}$ receiver.

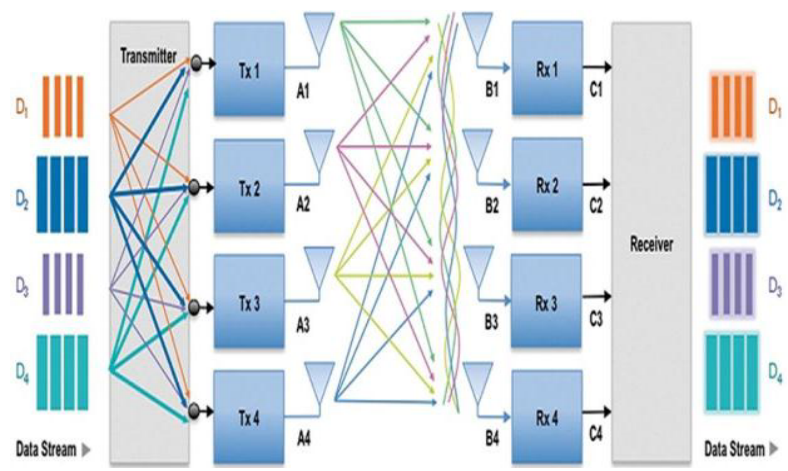

Figure 1. Block diagram of multiple input multiple output (MIMO) system[13].

\subsubsection{OFDM technique and system model}

Orthogonal Frequency Division Multiplexing (OFDM) is a form of broadband multicarrier modulation method that splits a high data rate signal stream into a multiple smaller sub-signals that are transmitted simultaneously at different frequencies and in this way, it is less sensitive to frequency selective fading. An OFDM signal consists of a number of closely spaced modulated carriers which are orthogonal to each other so as to avoid from signal interference between them. When the modulation of any form of information -video, voice, data, etc. is applied to a carrier, then sidebands spread out either side. Although the sidebands of each carriers overlap, the receiver can still receive them without interference because the modulated signals are orthogonal to each other. For this the carrier spacing is made equal to the reciprocal of the symbol period. OFDM reduces the amount of crosstalk in signal transmission.

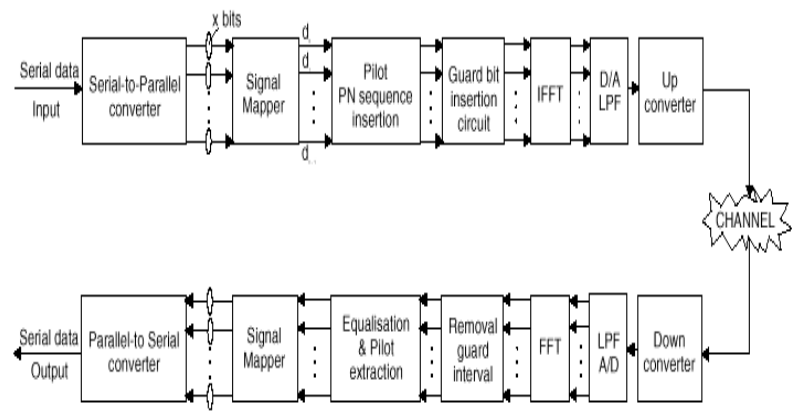

Figure 2. OFDM system configuration

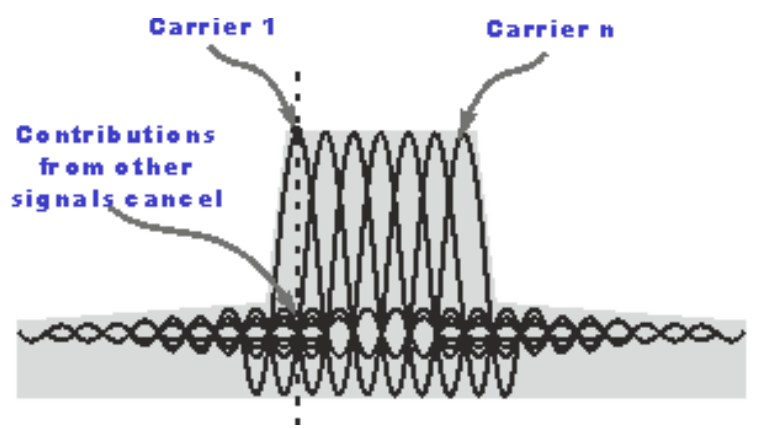

Figure 3. OFDM spectrum

The OFDM system converts the high speed serial data streams to low speed parallel streams that can be transmitted in $\mathrm{N}$ sub-channels. The signals are modulated by $\mathrm{N}$ sub-carriers which are orthogonal to each other and the modulated signals are sent together. One requirement of the OFDM transmitting and receiving system is that they must be linear. Any non-linearity will produce interference between the transmitted and received signals as a result of inter-modulation distortion. This will generate unwanted signals which would cause interference and weaken the orthogonality of the transmission. At the receiver, they are received with the relevant sub-carriers and restores the original high speed data by converting parallel to serial converter. The system configuration for OFDM is shown in fig.(2). 


\section{MIMO-OFDM wireless communication systems}

\subsection{Working methodology}

The simulation is carried out first by designing a MIMOOFDM system using GNU radio by directly connecting the blocks. Typically the system may have blocks of random source, OFDM modulator, noise source, channel model and OFDM demodulator to accomplish the data transfer. Main emphasis is given on measuring the Error Rate value (BER) by employing different types of modulation techniques. BER is a parameter which is used to represent the number of received bits that have been changed due to transmission impairments such as the interference, noise, distortion etc. It measures the whole end to end performance of the given system that includes the transmitter, receiver and the medium. It is defined as the rate in which error occur in the OFDM system. At the transmitter side, the system modulates the incoming signals into OFDM symbols and at the receiver side, it performs synchronization, FFT and demodulation of the incoming OFDM symbols and passes packets up to the higher layer. The Error Rate block calculates the BER or the SER(Symbol Error Rate). It compares the input byte streams to the reference byte streams. Each byte in the incoming stream represents one symbol. The bits per symbol parameter is useful for calculating the BER. The system considerations for the proposed work aresampling rate- $32 \mathrm{~K}$, packet length-96, FFT-512, occupied tones- 200, cyclic prefix length-128, channel typeAWGN channel.

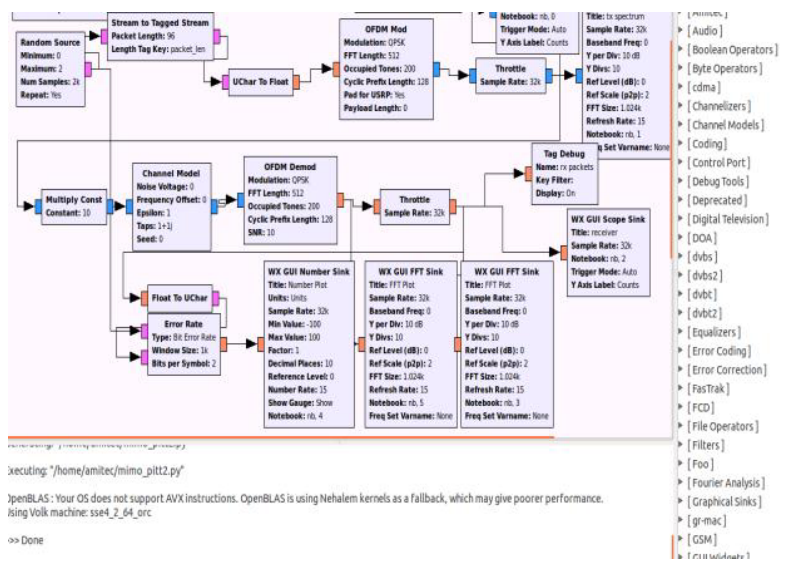

Figure 4. Designed GNU schematic for the OFDM MIMO system

\subsubsection{Result analysis and discussions}

The performance of the designed system is assessed by determining the data error rate during transmit and receive process. BER (bit error rate) is oftentimes used to measure the performance of digital communication system in the presence of noise, i.e. SNR (signal to noise ratio). The transmitted signal waveform and received data streams are obtained and are shown in Fig. (5) and (6) respectively. Table 1 shows the BER performance of the system for different modulation schemes.

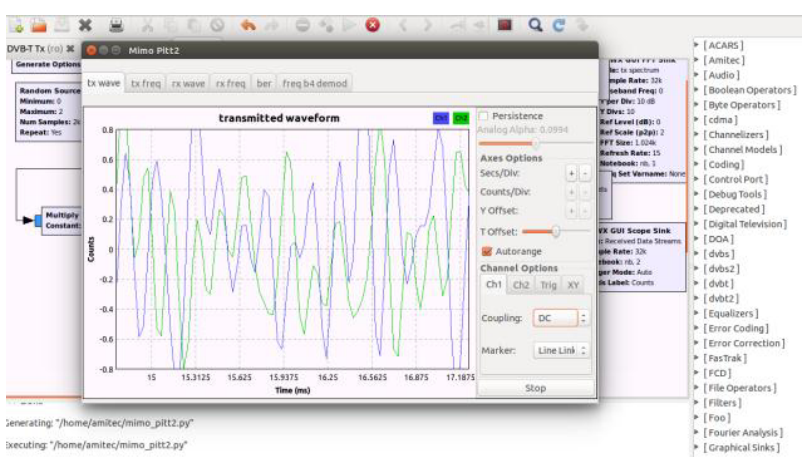

Figure 5. Transmitted waveform at the transmitter side

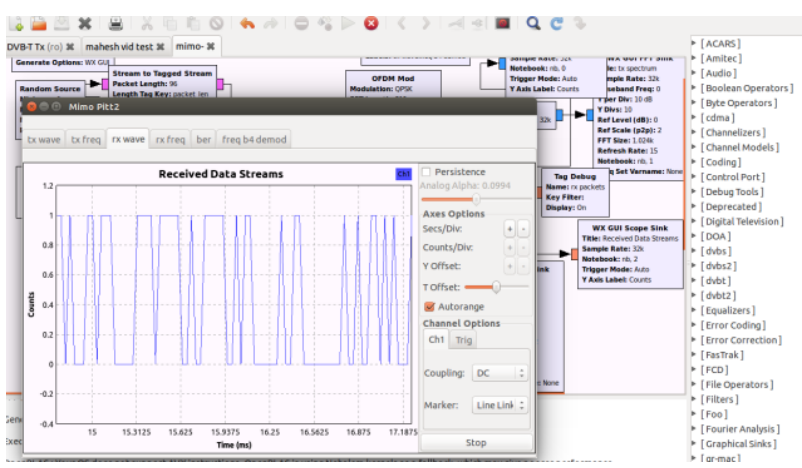

Figure 6. Received data streams at the receiver side

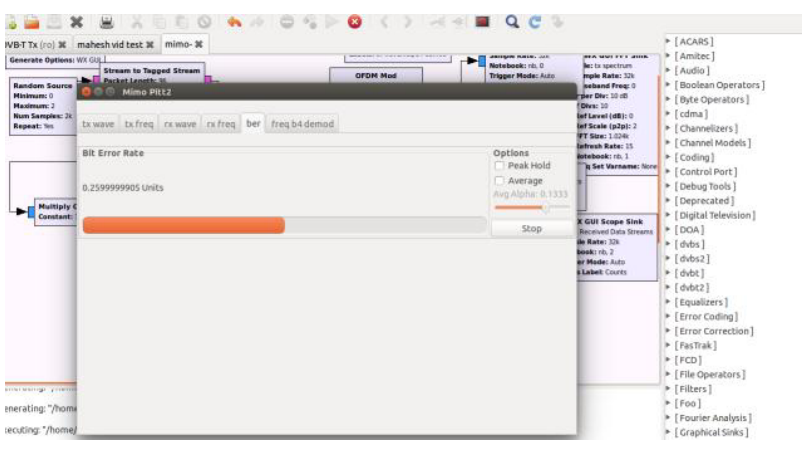

Figure 7. BER values generated

Table 1. BER for different modulation schemes.

\begin{tabular}{|c|c|l|l|l|l|}
\hline $\begin{array}{c}\text { SN } \\
\text { R(d } \\
\text { B) }\end{array}$ & BPSK & QPSK & QAM 16 & QAM 64 & 8PSK \\
\hline 0 & $\begin{array}{c}0.7960 \\
00\end{array}$ & $\begin{array}{l}0.801 \\
500\end{array}$ & $\begin{array}{l}0.812 \\
500\end{array}$ & $\begin{array}{l}0.808 \\
000\end{array}$ & $\begin{array}{l}0.797 \\
778\end{array}$ \\
\hline 2 & $\begin{array}{c}0.772 \\
382\end{array}$ & $\begin{array}{l}0.783 \\
990\end{array}$ & $\begin{array}{l}0.806 \\
000\end{array}$ & $\begin{array}{l}0.805 \\
963\end{array}$ & $\begin{array}{l}0.791 \\
388\end{array}$ \\
\hline 4 & $\begin{array}{c}0.745 \\
816\end{array}$ & $\begin{array}{l}0.763 \\
588\end{array}$ & $\begin{array}{l}0.799 \\
500\end{array}$ & $\begin{array}{l}0.791 \\
000\end{array}$ & $\begin{array}{l}0.765 \\
504\end{array}$ \\
\hline 6 & 0.736 & 0.754 & $\begin{array}{l}0.788 \\
883\end{array}$ & $\begin{array}{l}0.789 \\
325\end{array}$ & $\begin{array}{l}0.759 \\
929\end{array}$ \\
\hline 8 & 0.711 & 0.748 & 0.771 & 0.782 & 0.744 \\
\hline
\end{tabular}




\begin{tabular}{|l|l|l|l|l|l|}
\hline & 725 & 812 & 870 & 500 & 164 \\
\hline \multirow{3}{*}{10} & 0.690 & 0.731 & $\begin{array}{l}0.746 \\
000\end{array}$ & $\begin{array}{l}0.782 \\
500\end{array}$ & 0.738 \\
& 151 & 232 & 050 \\
\hline
\end{tabular}

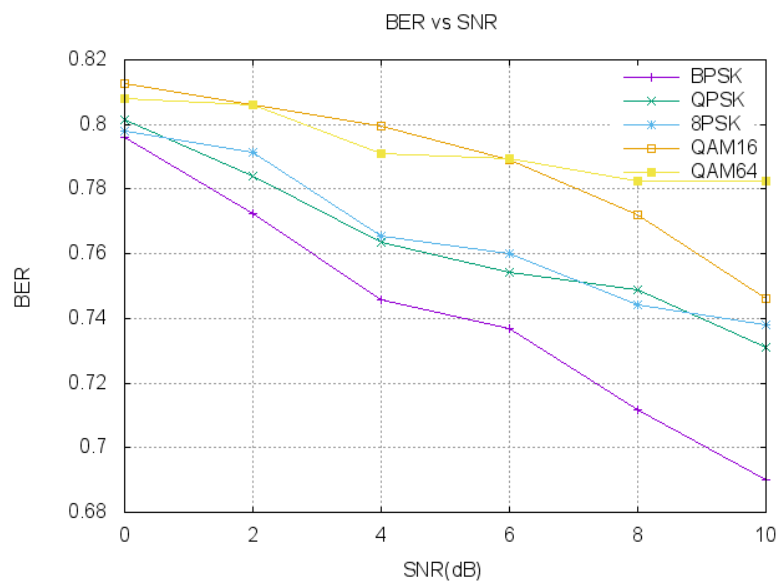

Figure 8. BER vs SNR curve of the MIMO-OFDM system

Table 1 shows the BER (Bit Error Rate) values as a function of varying SNR for the MIMO-OFDM system for different modulation schemes i.e. BPSK, QPSK, 8PSK, QAM16 and QAM64. Figure 8 shows the plot of the BER vs SNR. According to the results obtained and from the graph, it is seen that BER value depends on the number of bits per symbol and value of BER is low for BPSK which can be the best suitable modulation technique for information transmission in the OFDMMIMO. It is also seen that the BER greatly depends on SNR and with the increase of SNR, the BER decreases because a stronger noise increases BER and a stronger signal power decreases the BER.

\section{CONCLUSION}

In this paper, the performance of MIMO-OFDM system has been analysed with different modulations schemes. The BER values are obtained as a result with varying Signal to Noise Ratio (SNR) by putting different noise values at the channel model. Of the modulation schemes employed, BPSK is performing the best because of less bits per symbol it represents followed by QPSK scheme. The corresponding BER performance differs because they exhibit different tolerance margin to noise. Thus this lower modulation order reduces BER resulting in better and improved performance of the OFDM-MIMO system. Further works can be extended to the performance analysis of real time transmission and reception of the OFDM-MIMO system using USRPs which will be done under different environmental conditions and for different coding schemes.

\section{References}

1. J. G. Andrew, W. Choi, R. W. Heath jr., "Overcoming Interference in Spatial Multiplexing.." IEEE Wireless Communications, pp. 95-104, (2007)

2. Q. Li, G. Li, W. Lee, Z. Li, "MIMO techniques in WiMAX and LTE: a feature overview..." IEEE Communications Magazine, pp. 86-92, (2010)

3. H. Sampath, S. Talwar, J. Tellado, V. Erceg, A. Paulraj, "A Fourth Generation MIMO-OFDM Broadband Wireless System: Design, Performance, and Field Trial Results.." IEEE Communications Magazine, pp. 143-149, (2002)

4. A. Sharma, A. Garg, "BER Analysis Based on Transmit and Receive Diversity Techniques in MIMO-OFDM System" IJECT, pp. 58-60, (2012)

5. X. Li, W. Hu, H. Y'zadeh, A. Qureshi, "A Case Study of a MIMO SDR Implementation..” IEEE, pp. 1-7, (2008)

6. S. Trivedi, Md. S. Raeen, S.S. Pawar, “BER Analysis of MIMO-OFDM System using BPSK Modulation Scheme.." IJACR, pages 208-214, (2012)

7. A. Pauraj, D. A. Gore, R. U. Nabar, H. Bolcskei, “ $A n$ Overview of MIMO Communications- A Key to Gigabit Wireless.." IEEE, pp. 198-218, (2004)

8. Y. S. Cho, J. Kim, W. Y. Yang, C. G. Kang, “ MIMO-OFDM Wireless Communications" with $M A T L A B$ (John Wiley \& Sons, 2010)

9. GNURadio. http://gnuradio.org/.

10. S. Cui, A. J. Goldsmith, A. Bahari, "Energyefficiency of MIMO and Cooperative MIMO Techniques in Sensor Networks.." IEEE Journal on Selected Areas in Communications, pp. 1-10, (2004)

11. H. Bolcskei, "MIMO-OFDM Wireless systems: Basics, perspectives, and challenges.." IEEE Wireless Communications, ppl 31-37, (2006)

12. Q. H. Spencer, C. B. Peel, A. L. Swindlehurst, M. Haaredt, "An introduction to the multi-user MIMO downlink.." IEEE Communication Magazine, pp. 6067, (2004)

13. B. Reddy, B. Lakshmi, "BER Analysis with Adaptive Modulation Coding in MIMO-OFDM.." IJWMT, 4, 20-34, (2014) 